\title{
Efficacy and Safety of Cryoneurolysis for Treatment of Chronic Head Pain Secondary to Occipital Neuralgia: A Pilot Study
}

\author{
Eric Grigsby' \\ Richard Radnovich ${ }^{2}$ \\ Srinivas Nalamachu ${ }^{3}$ \\ 'Neurovations, Napa, CA, USA; ${ }^{2}$ Injury \\ Care Medical Center, Boise, ID, USA \\ ${ }^{3}$ Mid America PolyClinic, Overland Park, \\ KS, USA
}

Purpose: Treatment of chronic pain associated with occipital neuralgia $(\mathrm{ON})$ is complex, and no consensus statement or guidelines have been published for ON management. This pilot study evaluated the efficacy and safety of cryoneurolysis for management of ONassociated chronic pain.

Patients and Methods: The study was a prospective, multicenter, nonrandomized cohort study assessing the degree and duration of clinical effect of cryoneurolysis therapy for reducing pain in patients diagnosed with unilateral or bilateral ON. The primary outcome measure was improvement in pain due to ON from baseline to day 7, measured on an 11point numeric rating scale for pain. Secondary outcome measures included duration of treatment effects and safety events, including anticipated observations and adverse events. Treatment effect was assessed at days 7,30 , and 56 by asking the patient if they were continuing to experience a treatment effect, with potential responses of "effect," "no effect," or "no longer effective." A posttreatment questionnaire evaluated patient satisfaction.

Results: Twenty-six patients ( 9 men, 17 women) with a mean age of 49.1 years enrolled and completed the study. A total of $64 \%(16 / 25)$ of participants reported a clinically important improvement of $\geq 2$ points in numeric rating scale pain scores at day 7 ; similar results persisted to day 30 . Treatment effects were reported by $50 \%(13 / 26)$ of participants at day 30 , with a continued effect reported by $35 \%$ (9/26) of participants at day 56 . Overall, $\sim 70 \%$ of participants were satisfied with treatment at 7, 30, and 56 days. No serious anticipated observations, adverse events, or unanticipated adverse device effects were reported.

Conclusion: Cryoneurolysis provided significant relief from pain associated with $\mathrm{ON} \leq 30$ days after treatment and had an acceptable safety profile.

Keywords: cryoanalgesia, headache, peripheral neuropathic pain, cephalgia, migraine, pain management

\section{Plain Language Summary}

Occipital neuralgia $(\mathrm{ON})$ is a medical condition that may cause serious, stabbing headache. Healthcare providers use a variety of treatments for ON, but there remains a need for a longlasting therapy for $\mathrm{ON}$ that also does not require a major surgical procedure.

In a clinical trial with twenty-six patients, we studied the effect of a medical procedure called cryoneurolysis. This procedure works by freezing a specific nerve, which may help improve pain. We studied if cryoneurolysis can improve pain due to ON a week after receiving treatment. We also examined if this procedure had any side effects.

Sixteen out of twenty-five patients $(64 \%)$ had less pain a week after treatment, and similar results were also observed after a month. Nine out of twenty-six patients $(35 \%)$
Correspondence: Eric Grigsby

3416 Valle Verde Drive, Napa, CA, 94558

USA

Tel + I 707-252-9656

Fax + I 707-258-2780

Email eg@neurovations.com 
continued to see a positive effect from the therapy approximately two months from the treatment. No serious side effects were reported. Approximately two months from the treatment, twentyone of twenty-six patients (81\%) said they would recommend the treatment to a family member and eighteen of twenty-six patients (69\%) said they would be willing to have the treatment again.

In summary, we have observed that cryoneurolysis can provide meaningful pain relief in patients with $\mathrm{ON}$ for up to a month, and cryoneurolysis appears to be safe.

\section{Introduction}

Occipital neuralgia $(\mathrm{ON})$ is a cause of headache that originates at the base of the head and spreads to the back, front, or side of the head, including the area behind the eyes. ${ }^{1,2} \mathrm{ON}$ can be unilateral or bilateral and is characterized by paroxysmal severe pain that is shooting, stabbing, or sharp in quality in the distribution of the greater or lesser occipital nerves. ${ }^{1}$ The etiology of ON may be idiopathic or structural and can vary from patient to patient. ${ }^{3}$ For many patients, the etiology is unknown, but some events associated with the development of ON include irritation, inflammation, compression, or injury of the occipital nerves. ${ }^{3,4}$

According to the International Headache Society diagnostic criteria for $\mathrm{ON}$, diagnosis can be confirmed on the basis of clinical presentation of pain (ie, episodic stabbing pain and tenderness over the affected nerve) and a temporary improvement in pain with local anesthetic block of the nerve. ${ }^{1,4}$ However, occipital nerve block is not a specific diagnostic criterion for $\mathrm{ON}$, and falsepositive results can occur with cases of migraines and cluster headaches. ${ }^{4}$ In addition, patients with ON may experience symptoms similar to migraine or cluster headaches, such as photophobia, phonophobia, dizziness, nausea and vomiting, and posterior scalp paresthesia, further complicating diagnosis. ${ }^{2,3} \mathrm{ON}$ is often misdiagnosed as migraine, fibromyalgia, cervical spine arthritis, cervical disc disease, and cervicogenic headache. ${ }^{3,5}$

In addition to diagnostic difficulty, management of ON can be complex, and there is currently no consensus treatment for ON. ${ }^{4}$ Management of ON typically starts with physical measures such as posture correction, antiinflammatory medications, medications effective for neuropathic pain (eg, anticonvulsants, antidepressants), and opioids. Additional therapeutic approaches include botulinum toxin, local nerve blocks and corticosteroid injections, radiofrequency ablation, and neurostimulation. In difficult-to-treat cases with presumed nerve entrapment, surgical decompression is considered. ${ }^{3,4}$ Each strategy offers varying relief of symptoms, duration of action, and invasiveness. However, there remains a need for novel nonsurgical, minimally invasive, and long-lasting approaches to ON pain management. ${ }^{6}$

Cryoneurolysis is a form of thermal neurolysis involving the application of cold temperature to peripheral sensory nerves to achieve a long-lasting nerve block. When nitrous oxide is used, cryoneurolysis occurs at temperatures of $-20^{\circ} \mathrm{C}$ to $-100^{\circ} \mathrm{C}{ }^{7}$ In this temperature range, the affected nerve will undergo Wallerian degeneration, in which the nerve axon is targeted but the nerve sheath is preserved, allowing for axonal regeneration. ${ }^{7-9}$ Advantages of cryoneurolysis over neurolytic modalities such as chemical neurolysis and radiofrequency ablation include less damage to tissue architecture and adjacent tissues and a reduced risk of neuroma. ${ }^{9-11}$ Although cryoneurolysis has been previously investigated for cervicogenic headache, ${ }^{12}$ studies evaluating the potential benefit and safety of cryoneurolysis for treatment of chronic head pain secondary to $\mathrm{ON}$ are needed.

The investigated cryoneurolysis device in the current study was precursor to a class 2 medical device cleared by the US Food and Drug Administration (Pacira Cryotech, Fremont, CA).${ }^{13}$ Both devices deliver liquid nitrous oxide within a closed-end probe to apply freezing temperatures as low as $-88^{\circ} \mathrm{C}$ to target nerves, resulting in reversible Wallerian degeneration. ${ }^{7}$ In addition, both systems include an integrated skin warmer, which may protect the skin and hair in the treatment area from subdermal or follicular necrosis. ${ }^{7}$ Previous results from a multicenter, randomized, double-blind, sham-controlled study demonstrated that cryoneurolysis via the newer-generation device was a safe and effective approach to treating pain and symptoms associated with osteoarthritis of the knee. ${ }^{14}$ Other studies have suggested that cryoneurolysis via the newer-generation device prior to total knee arthroplasty can reduce knee symptoms, shorten length of hospital stay, and reduce opioid consumption compared with standard of care. ${ }^{15,16}$

Use of cryoneurolysis or cryoablation (which uses colder temperatures than cryoneurolysis) to treat unilateral or bilateral occipital neuralgia with other devices has been described previously. ${ }^{2,17,18}$ However, additional safety and efficacy data are required to validate the approach more using the moderate temperature range produced by nitrous oxide, which enables nerve regeneration. This was a proofof-concept pilot study designed to provide initial evaluation of the efficacy, safety, and duration of clinical effect 
with cryoneurolysis in patients with $\mathrm{ON}$, which may help inform the direction of future controlled studies.

\section{Patients and Methods Study Design and Population}

This was a prospective, multicenter, single-arm, pilot study to assess the safety and effectiveness of cryoneurolysis for pain due to ON (NCT01753765). Patients were treated between November 2012 and December 2013. Individuals were eligible for inclusion in the study if they were aged $\geq 18$ years, had a confirmed diagnosis of unilateral or bilateral $\mathrm{ON}$, had a mean numeric rating scale (NRS) pain score of $\geq 4$ during the 7 days leading up to the study, and were not pregnant at the time of treatment; patients on medication before starting treatment were required to maintain a stable medication schedule, as determined by the physician, prior to treatment. Individuals were excluded if they had a current diagnosis of fibromyalgia, chronic back pain, or chronic migraines; history of cerebrovascular accident, head trauma, stroke, or bone deformity; severe pain for any reason other than $\mathrm{ON}$; any clotting disorder and/or use of anticoagulant within 7 days prior to start of treatment; injections for pain relief or neuromodulation to the upper trunk or head within 3 months prior to start of treatment; anesthetics or steroids within 30 days prior to start of treatment; or previous surgery in intended area of treatment.

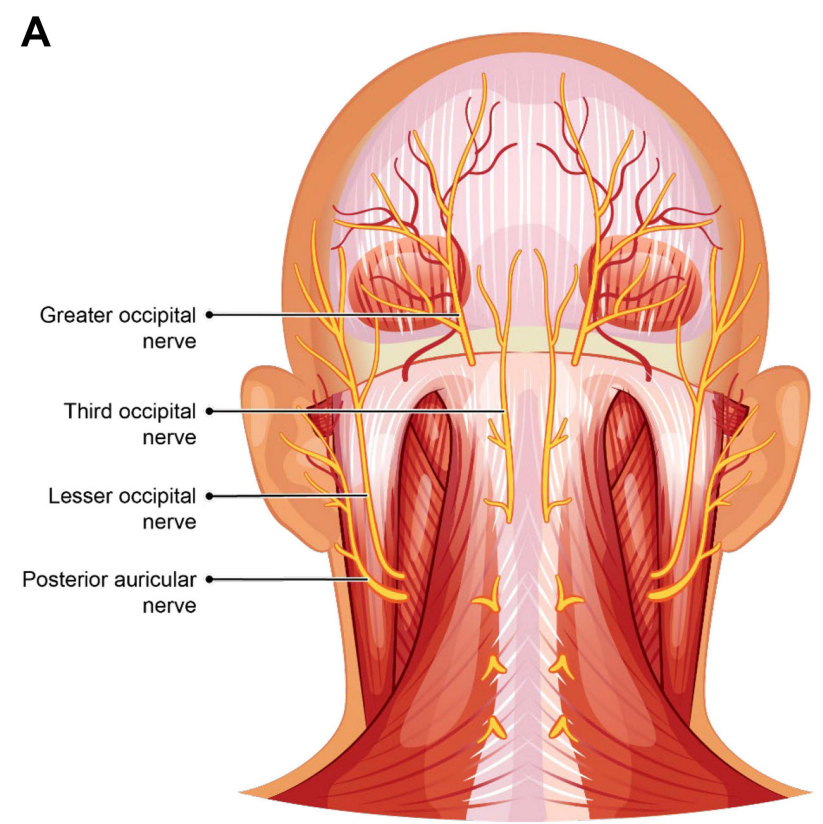

\section{Procedures and Treatments}

The treatment target was the greater occipital nerve (Figure 1). Because the anatomical landmark technique used for identifying the treatment area could not reliably differentiate the greater occipital nerve from the lesser occipital or the third occipital nerves, any of these nerves may have been targeted by treatment. The nerves were accessed by locating adjacent landmarks with ultrasound and/or percutaneous nerve stimulation. Local cutaneous anesthesia was administered via subdermal injection of local anesthetic. Treatment was performed by inserting the cryoprobe in a linear fashion, with subsequent treatments to block the nerve path at the location identified by the investigator. Each treatment cycle consisted of a 15second pre-warming cycle, a 60 -second treatment delivery, and a 10-second warming period after treatment. After the treatment cycle was completed, the cryoprobe was removed and reinserted to form a treatment line intersecting the predicted nerve path. Patients with a bilateral indication could receive treatment unilaterally or bilaterally at the discretion of the investigator.

\section{End Points and Assessments}

The primary outcome measure of the study was improvement from baseline in pain due to $\mathrm{ON}$ at day 7 as measured on an 11-point (scale of 0 to 10) NRS for pain, where 0 represents no pain and 10 represents very severe pain. Pain NRS scores were also assessed before

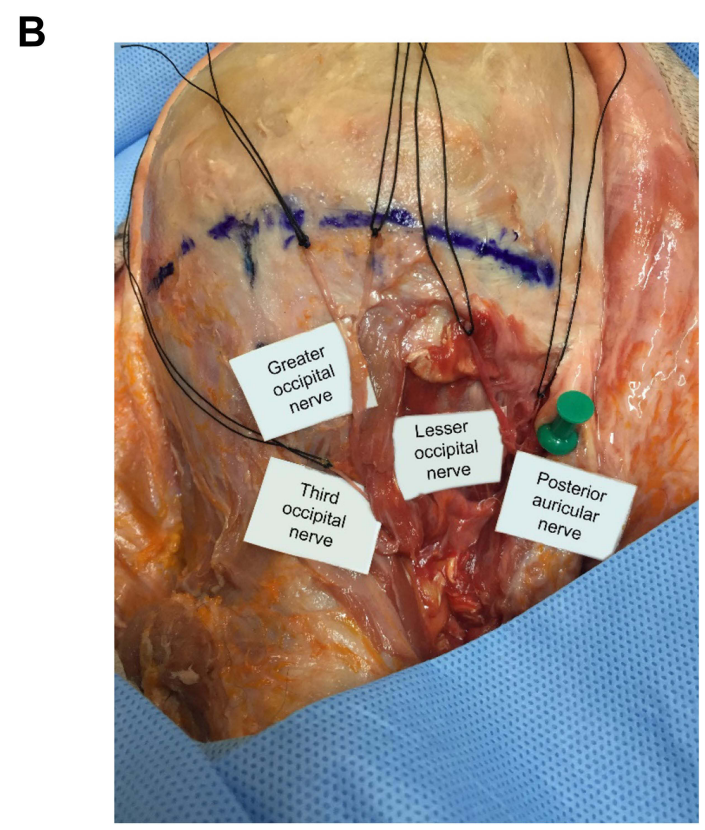

Figure I Nerve map of intended treatment area, including greater occipital nerve and lesser occipital nerve (A), and anatomical dissections of the treatment area (B). 
treatment, immediately post treatment, and at 30 days post treatment.

Secondary endpoints included duration of treatment effects and safety, including adverse events (AEs). Duration of treatment effect was assessed at days 7, 30, and 56 by asking if patients were continuing to experience a treatment effect; potential responses included "effect," "no effect," or "no longer effective." Patients who reported a treatment effect at day 56 were monitored via phone follow-up at 4-week intervals up to day 112 for as long as they were experiencing a treatment effect. This assessment was also completed via phone at days 84 and 112, and at 4-week intervals thereafter.

On the basis of known risks associated with cryoneurolysis devices, several treatment-related AEs (TRAEs) were defined a priori and reported. Predefined TRAEs were bruising, tingling, erythema, swelling, itching, local pain/tenderness, erosion/ulceration, crusting, dimpling, hyperpigmentation, and hypopigmentation. These anticipated TRAEs do not typically require medical intervention and are transient in duration. Any AE that exceeded the expected response to treatment in severity or in duration was separately reported. To assess safety, the treatment area was examined during each visit for TRAEs. All AEs were reported. AEs, serious AEs (SAEs), and unanticipated adverse device effects (UADEs) were also assessed at all visits.

To assess patient satisfaction, a posttreatment questionnaire was administered that included 4 yes/no questions: one question regarding whether the patient would recommend the treatment to a family member, one regarding whether the patient would take the treatment again, one regarding the occurrence of anticipated observations (ie, safety signals), and one regarding pain from study treatment. If the patient answered "yes" to the occurrence of anticipated observations and/or pain, he or she was asked to report the impact of anticipated observations on their daily routine and/or determine the severity of pain from study treatment on a scale of 1 to 5 .

This postmarketing study was approved by an Institutional Review Board (IntegReview Ethical Review Board, now Advarra, Columbia, MD, USA) and was conducted in accordance with the general principles set forth in the International Council for Harmonisation Guidelines for Good Clinical Practice and the Declaration of Helsinki. All patients provided written informed consent before enrollment in the study.

\section{Statistical Analysis}

NRS pain scores were analyzed for response rates, clinically important differences, and statistically significant improvements from baseline (ie, preprocedure day 0 ) at each follow-up visit. NRS pain score changes from baseline were calculated by subtracting the score at posttreatment, Day 7, and Day 30 from the NRS pain score reported at baseline, and these differences were analyzed for statistical significance using a null hypothesis (ie, difference from baseline of 0 ). A paired, two-tailed $t$-test was used to account for the possibility of patients worsening over the course of the study and had a significance level of $P<0.05$. Duration of treatment effect was analyzed for the number of responders at each follow-up point.

\section{Results}

\section{Patient Disposition and Baseline Demographics}

Overall, 26 patients were enrolled in the study, including 17 patients who received bilateral treatment and 9 patients who received unilateral treatment, for a total of 43 treatment areas. All patients completed the study and were included in the final analysis. The majority of the patients $(\mathrm{n}=17 ; 65 \%)$ in the study were female, with a mean (standard deviation [SD]) age of 49.1 (13.0) years and mean (SD) baseline NRS pain score of $6.3(1.5)$.

\section{Efficacy NRS Pain Scores}

NRS pain scores decreased immediately after treatment and remained lower than baseline scores through at least day 30 (Supplemental Figure). For the primary outcome measure, $84 \%$ of patients $(21 / 25)$ reported $a \geq 1$ point improvement in NRS pain scores at day 7 . When NRS pain scores were assessed immediately post treatment and at day 30, 92\% (24/26) and $78 \%(18 / 23)$ of patients reported a $\geq 1$ point improvement, respectively. A clinically important improvement in NRS pain scores has been previously defined as an improvement of 1.3 on a scale of 0 to $10 .{ }^{19}$ Because this study only included NRS responses on a whole-number scale, an improvement of $\geq 2$ points was considered clinically meaningful, which was experienced by $88 \%$ (23/26), $64 \%$ $(16 / 25)$, and $74 \%$ (17/23) of patients immediately post treatment, at day 7, and at day 30, respectively. Moreover, mean (SD) NRS improvements of 2.8 (2.1), 3.8 (2.3), and 3.6 (2.4) exceeded the clinically important threshold immediately posttreatment, at day 7 , and at day 30 , respectively (Table 1 ). 
Table I Mean Improvement in NRS from Baseline

\begin{tabular}{|l|c|c|c|c|}
\hline Parameter & Baseline (n=26) & Posttreatment (n=26) & Day $7(\mathbf{n = 2 5})^{\mathbf{a}}$ & Day 30 (n=23) \\
\hline Mean (SD) NRS score & $6.3(1.5)$ & $2.8(2.1)$ & $3.8(2.3)$ & $3.6(2.4)$ \\
Mean (SD) point improvement & - & $3.5(2.2)$ & $2.5(2.5)$ & $2.7(2.2)$ \\
Mean (SD), \% improvement & - & $55(31)$ & $35(42 \%)$ & $43(35)$ \\
$P$ value (change from baseline) & - & $<0.0001$ & $<0.0001$ & $<0.0001$ \\
\hline
\end{tabular}

Note: ${ }^{a}$ NRS was not assessed for I patient at day 7.

Abbreviations: NRS, numeric rating scale; SD, standard deviation.

\section{Duration of Treatment Effect}

The proportion of patients reporting a treatment effect at each follow-up was calculated both as a proportion of the total study population and as a proportion of the patients with data at each respective follow-up visit (Table 2). At day $30,50 \%$ of patients (13/26) reported a continued effect from treatment, and at day 56, 35\% (9/26) reported a continued effect. Treatment effects were reported at up to 112 days after cryoneurolysis in some patients.

\section{Patient Experience}

The responses to the questionnaire on patient satisfaction are shown in Table 3. At day 7,69\% of patients (18/26) indicated that they would recommend the treatment to a family member; this number increased to $81 \%(21 / 26)$ at day 56 . When asked whether they would be willing to have the treatment again, $69 \%$ of patients $(18 / 26)$ responded "yes" at days 7 and 56 .

A total of $54 \%(14 / 26), 4 \%(1 / 23)$, and 0 patients reported treatment-related pain on days 7,30 , and 56 , respectively. Patients who reported pain were asked to rate the pain on a scale of 1 to 5 ( 1 = not at all painful, $5=$ very painful $)$; only one patient at day 7 reported treatment-related pain as a 4 , and all other pain scores were $\leq 3$.

\section{Safety}

\section{Anticipated Treatment-Related Adverse Events}

At day 7, 77\% (20/26) of patients reported anticipated TRAEs, but this proportion decreased at subsequent follow-up visits (Table 4). Most patients who reported any
Table 3 Patient Satisfaction

\begin{tabular}{|l|l|l|l|}
\hline Parameter, n/N (\%) & Day 7 & Day 30 & Day 56 \\
\hline $\begin{array}{l}\text { Would recommend to } \\
\text { family member }\end{array}$ & $18 / 26(69)$ & $19 / 23(83)$ & $21 / 26(81)$ \\
\hline $\begin{array}{l}\text { Would have treatment } \\
\text { again }\end{array}$ & $18 / 26(69)$ & $16 / 23(70)$ & $18 / 26(69)$ \\
\hline
\end{tabular}

anticipated TRAEs indicated these events had little to no impact on their daily routine.

Anticipated TRAEs were assessed per treatment area across all patients (Table 5). No severe anticipated TRAEs were reported in the study. The most frequently reported anticipated TRAE was crusting at the insertion site. Crusting was reported as mild in 23/43 (53\%) treatment areas and moderate in $4 / 43(9 \%)$ treatment areas at day 7 ; all crusting resolved before the assessment on day 30. Redness/inflammation, local pain, swelling, itching, and hyperpigmentation were all reported in over $20 \%$ of the study population at day 7. Twelve percent of patients reported mild tingling at day 7 , and all cases of tingling resolved by day 30 ; however, 2 additional reports of tingling were reported at day 56 in 2 patients who had not previously reported any tingling. Anticipated TRAEs were less prevalent beyond day 7; redness/inflammation, local pain, itching, hyperpigmentation, and tingling were reported in $<10 \%$ of patients on days 30 and 56. No new cryoneurolysis treatment risks were documented in the study population.

A total of 6 AEs reported by 5 patients (19\%) were unanticipated in either type, severity, or duration, of which

Table 2 Patients Reporting Continued Treatment Effect Over Initial Follow-Up Period

\begin{tabular}{|l|c|c|c|c|c|}
\hline Population & Day 7 & Day 30 & Day 56 & Day 84 & Day II2 \\
\hline Patients with treatment effect in study population, n/N (\%) & $16 / 26(62)$ & $13 / 26(50)$ & $9 / 26(35)$ & $4 / 26(15)$ & $3 / 26(12)$ \\
Patients with treatment effect in completed population, ${ }^{\mathrm{a}} \mathrm{n} / \mathrm{N}(\%)$ & $16 / 26(62)$ & $13 / 23(57)$ & $9 / 2 \mathrm{I}(43)^{\mathrm{b}}$ & $4 / 8(50)$ & $3 / 4(75)$ \\
\hline
\end{tabular}

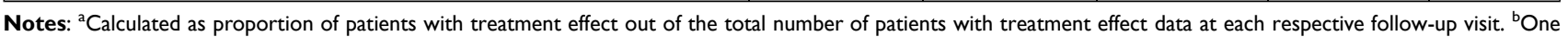
study site (including 5 patients) did not assess treatment effect at the day 56 follow-up visit. 
Table 4 Patient-Reported Anticipated TRAEs

\begin{tabular}{|l|c|c|c|}
\hline Parameter & Day 7 & Day 30 & Day 56 \\
\hline Patients with any anticipated TRAE, n/N (\%) & $20 / 26(77)$ & $4 / 23(17)$ & $3 / 26(12)$ \\
Impact of anticipated TRAE on daily routine ${ }^{\mathrm{a}}$ & & & $0 / 3(0)$ \\
I (very negative impact) & $0 / 20(0)$ & $0 / 4(0)$ & $0 / 3(0)$ \\
2 & $1 / 20(5)$ & $1 / 4(25)$ & $1 / 3(33)$ \\
3 & $4 / 20(20)$ & $0 / 4(0)$ & $0 / 0(0)$ \\
4 & $6 / 20(30)$ & $3 / 4(75)$ & $2 / 3(67)$ \\
5 (no impact at all) & $9 / 20(45)$ & $0 / 4(0)$ & \\
\hline
\end{tabular}

Note: ${ }^{a}$ Only includes patients who reported any anticipated TRAEs.

Abbreviation: TRAE, treatment-related adverse event.

2 AEs (pain in treatment area and increased pain in neck area) were considered possibly treatment related. Both events were considered moderate in severity and did not require an intervention. No serious AEs, discontinuations due to $\mathrm{AE}$, or unanticipated adverse device effects were reported during the study.

\section{Discussion}

Cryoneurolysis provided rapid and meaningful relief from pain associated with ON. A total of $88 \%$ of patients experienced clinically important improvement in pain scores $(\geq 2$ points) on the day of the treatment. The NRS scores also significantly improved from baseline by mean 3.5 points on the day of treatment, representing an average of a $55 \%$ improvement in pain scores from baseline. Significant reductions in pain scores were consistently observed through day 30 , and the mean reduction in NRS score exceeded the clinically important improvement threshold at day 30 . The effects of cryoneurolysis lasted up to 8 weeks in $35 \%$ of patients.

Diagnostic challenges due to resemblance of ON manifestations to other disorders ${ }^{20}$ and lack of consensus guidelines make managing ON difficult. Over-the-counter pain medications have short-term effects on pain associated with $\mathrm{ON}$ and may have increased risks with longterm use. ${ }^{4,21}$ Occipital nerve blocks using local anesthetics with or without steroids can produce short-term relief of symptoms, but their limited duration of action may restrict their use as long-term management. ${ }^{4}$ Although botulinum toxin has had success in management of migraines, ${ }^{22}$ use of botulinum toxin to manage pain associated with ON has not been consistently helpful. ${ }^{4}$ Pulsed radiofrequency treatment has demonstrated short- to intermediate-term pain control for patients with $\mathrm{ON},{ }^{4}$ but important prospective evidence on the effectiveness of pulsed radiofrequency treatment is lacking. More recently, neurostimulation has been shown to provide symptom relief in patients with $\mathrm{ON}$, but it requires an implanted electrode and, in most cases, an implanted pulse generator for long-term use. ${ }^{4,23}$ Use of cryoneurolysis within the moderate temperature range produced by nitrous oxide to manage pain associated with ON could provide a minimally invasive, effective, and safe alternative for

Table 5 Reported Anticipated TRAEs by Treatment Area

\begin{tabular}{|c|c|c|c|c|c|c|c|c|c|}
\hline \multirow[t]{2}{*}{ Anticipated TRAE, n (\%) } & \multicolumn{3}{|c|}{ Day $7(N=43)$} & \multicolumn{3}{|c|}{ Day $30(\mathrm{~N}=37)$} & \multicolumn{3}{|c|}{ Day $56(\mathrm{~N}=29)^{\mathrm{c}}$} \\
\hline & Mild & Moderate & Severe & Mild & Moderate & Severe & Mild & Moderate & Severe \\
\hline Crusting & $23(53)$ & $4(9)$ & $0(0)$ & $0(0)$ & $0(0)$ & $0(0)$ & $0(0)$ & $0(0)$ & $0(0)$ \\
\hline Erythema & $17(43)$ & $3(7)$ & $0(0)$ & $2(5)$ & $0(0)$ & $0(0)$ & $0(0)$ & $0(0)$ & $0(0)$ \\
\hline Local pain & $12(28)$ & $5(12)$ & $0(0)$ & $2(5)$ & $0(0)$ & $0(0)$ & I (3) & $0(0)$ & $0(0)$ \\
\hline Swelling & II (26) & I (2) & $0(0)$ & $0(0)$ & $0(0)$ & $0(0)$ & $0(0)$ & $0(0)$ & $0(0)$ \\
\hline Itching & $9(2 \mathrm{I})$ & $0(0)$ & $0(0)$ & I (3) & $0(0)$ & $0(0)$ & $2(7)$ & $0(0)$ & $0(0)$ \\
\hline Hyperpigmentation & $8(19)$ & I (2) & $0(0)$ & $2(5)$ & $0(0)$ & $0(0)$ & $0(0)$ & $0(0)$ & $0(0)$ \\
\hline Bruising & $5(12)$ & $2(5)$ & $0(0)$ & $0(0)$ & $0(0)$ & $0(0)$ & $0(0)$ & $0(0)$ & $0(0)$ \\
\hline Tingling & $5(\mid 2)$ & $0(0)$ & $0(0)$ & $0(0)$ & $0(0)$ & $0(0)$ & $2(7)$ & $0(0)$ & $0(0)$ \\
\hline
\end{tabular}

Notes: ancludes 26 patients with a total of 43 treatment areas. ${ }^{b}$ Hypopigmentation, dimpling, and erosion were not reported by any patient at any follow-up visit. ${ }^{c}$ Anticipated TRAEs were not assessed at day 56 for 8 patients ( 14 total treatment areas).

Abbreviation: TRAE, treatment-related adverse event. 
these patients. Overall, this pilot study found cryoneurolysis therapy to be safe and tolerable, with no reported serious AEs, device-related serious AEs, or new cryoneurolysis-related risks to patients.

The favorable safety profile of cryoneurolysis may enable repeated treatments for chronic pain without increasing risks for patients; however, utility of repeated treatment requires further study. Cryoneurolysis is not expected to have a permanent effect on pain, as the $-88^{\circ} \mathrm{C}$ temperature achieved by the probe investigated here causes degeneration of the axon yet preserves the nerve sheath, allowing the targeted nerve to regenerate and regain function. ${ }^{7}$ Multiple cryoneurolysis treatments with the device investigated in this study have demonstrated nerve regeneration that is consistent and predictable, ${ }^{9}$ extending the potential clinical benefits of this therapy. However, while the NRS pain scores reported in this study were significantly reduced compared with baseline scores $(\mathrm{P}<0.0001)$, pain was not completely abolished (ie, NRS pain scores did not reach 0 ). The significance of this result is beyond the scope of the current study, and future studies may help determine the optimal approach for management of ONassociated pain.

There were several limitations to this study. The main limitation of this study was its uncontrolled, unblinded design, which precludes a comparison of the investigated treatment with other $\mathrm{ON}$ treatments. Although this was a prospective study, the lack of a control group likens this pilot study to a case series and introduces potential for bias. Thus, the efficacy reported in this report should be interpreted carefully. In addition, the small population size included in this study limits the generalizability of these findings. Finally, this study did not include outcome measures to assess the impact of cryoneurolysis on participants' quality of life (eg, PQRST, QISS TAPED). However, these limitations do not preclude the utility of this preliminary study in informing future, more rigorous, clinical trials. While any conclusions drawn from this pilot study must be limited, the results provide foundational knowledge on the degree and duration of cryoneurolysis effect to support larger, controlled studies of this treatment in patients with ON. Future clinical studies of cryoneurolysis for treatment of chronic head pain secondary to ON should include a comparator group (eg, placebo or sham procedure, or active control of another ON treatment); a randomized design; more thorough characterization of the participant population at baseline, including the duration of chronic pain associated with ON; and comparisons of both NRS pain scores and quality-of-life measures (eg, PQRST, QISS TAPED) between treatment groups.

In the majority of patients in this study, cryoneurolysis therapy provided fast-acting and durable pain management due to $\mathrm{ON}$, and no serious AEs were reported. The efficacy and safety observed in this pilot study support further investigation of cryoneurolysis for relief of chronic pain associated with ON.

\section{Data Sharing Statement}

The data sets used and/or analyzed during the current study are available from the corresponding author upon reasonable request.

\section{Acknowledgments}

This study was supported by Pacira BioSciences, Inc. Writing and editorial assistance were provided under the direction of the authors by Elizabeth Harvie, PhD, CMPP, ELS, and Emilia Raszkiewicz of MedThink SciCom with support from Pacira BioSciences, Inc.

\section{Author Contributions}

All listed authors meet the criteria for authorship set forth by the International Committee of Medical Journal Editors, ie, they made a significant contribution to the work reported. All authors made substantial contributions to conception and design, acquisition of data, or analysis and interpretation of data; took part in drafting the article or revising it critically for important intellectual content; agreed to submit to the current journal; gave final approval of the version to be published; and agree to be accountable for all aspects of the work.

\section{Funding}

This study was sponsored by Myoscience Inc., including study design and funding. Myoscience Inc. was acquired by Pacira BioSciences, Inc. after completion of the study. Pacira BioSciences, Inc. was involved in the analysis and interpretation of the data. Writing and editorial assistance were also supported by Pacira BioSciences, Inc.

\section{Disclosure}

Dr Richard Radnovich reports grants from Myoscience, during the conduct of the study. The authors report no other conflicts of interest in this work. 


\section{References}

1. Headache Classification Committee of the International Headache Society (IHS). The International Classification of Headache Disorders, 3rd edition. Cephalalgia. 2018;38(1):1-211. doi:10.1177/ 0333102417738202

2. Stogicza A, Trescot A, Rabago D. New technique for cryoneuroablation of the proximal greater occipital nerve. Pain Pract. 2019;19 (6):594-601. doi:10.1111/papr.12779

3. Barna S, Hashimi M. Occipital neuralgia. Pain Manag Rounds. 2004;1(7):1-5.

4. Choi I, Jeon SR. Neuralgias of the head: occipital neuralgia. $J$ Korean Med Sci. 2016;31(4):479-488. doi:10.3346/jkms.2016.31.4.479

5. Yi X, Cook AJ, Hamill-Ruth RJ, Rowlingson JC. Cervicogenic headache in patients with presumed migraine: missed diagnosis or misdiagnosis? $J$ Pain. 2005;6(10):700-703. doi:10.1016/j. jpain.2005.04.005

6. Urits I, Schwartz RH, Patel P, et al. A review of the recent findings in minimally invasive treatment options for the management of occipital neuralgia. Neurol Ther. 2020;9(2):229-241. doi:10.1007/s40120-02000197-1

7. Ilfeld BM, Preciado J, Trescot AM. Novel cryoneurolysis device for the treatment of sensory and motor peripheral nerves. Expert Rev Med Devices. 2016;13(8):713-725. doi:10.1080/17434440.2016.1204229

8. Evans PJ. Cryoanalgesia. The application of low temperatures to nerves to produce anaesthesia or analgesia. Anaesthesia. 1981;36 (11):1003-1013. doi:10.1111/j.1365-2044.1981.tb08673.x

9. Hsu M, Stevenson FF. Wallerian degeneration and recovery of motor nerves after multiple focused cold therapies. Muscle Nerve. 2015;51 (2):268-275. doi:10.1002/mus.24306

10. Choi EJ, Choi YM, Jang EJ, Kim JY, Kim TK, Kim KH. Neural ablation and regeneration in pain practice. Korean J Pain. 2016;29 (1):3-11. doi:10.3344/kjp.2016.29.1.3

11. Brace C. Thermal tumor ablation in clinical use. IEEE Pulse. 2011;2 (5):28-38. doi:10.1109/MPUL.2011.942603

12. Kvarstein G, Högström H, Allen SM, Rosland JH. Cryoneurolysis for cervicogenic headache - a double blinded randomized controlled study. Scand J Pain. 2019;20(1):39-50. doi:10.1515/sjpain-20190086

13. Myoscience Inc. Iovera ${ }^{\circ}$ User Guide. Fremont, Ca: Myoscience, Inc.; 2018.
14. Radnovich R, Scott D, Patel AT, et al. Cryoneurolysis to treat the pain and symptoms of knee osteoarthritis: a multicenter, randomized, double-blind, sham-controlled trial. Osteoarthritis Cartilage. 2017;25(8):1247-1256. doi:10.1016/j.joca.2017.03.006

15. Dasa V, Lensing G, Parsons M, Harris J, Volaufova J, Bliss R. Percutaneous freezing of sensory nerves prior to total knee arthroplasty. Knee. 2016;23(3):523-528. doi:10.1016/j. knee.2016.01.011

16. Mihalko WM, Kerkhof A, Ford MC, Crockarell JR Jr, Harkess JW, Guyton JL. Cryoneurolysis before total knee arthroplasty in patients with severe osteoarthritis for reduction of postoperative pain and opioid use in a single-center randomized controlled trial. J Arthroplasty. 2020;36(5):1590-1598. doi:10.1016/j. arth.2020.11.013

17. Kim CH, Hu W, Gao J, Dragan K, Whealton T, Julian C. Cryoablation for the treatment of occipital neuralgia. Pain Physician. 2015;18(3):E363-368. doi:10.36076/ppj.2015/18/E363

18. Kastler A, Attyé A, Maindet C, et al. Greater occipital nerve cryoneurolysis in the management of intractable occipital neuralgia. J Neuroradiol. 2018;45(6):386-390. doi:10.1016/j. neurad.2017.11.002

19. Bijur PE, Latimer CT, Gallagher EJ. Validation of a verbally administered numerical rating scale of acute pain for use in the emergency department. Acad Emerg Med. 2003;10(4):390-392. doi:10.1111/ j.1553-2712.2003.tb01355.x

20. Hoppenfeld JD. Cervical facet arthropathy and occipital neuralgia: headache culprits. Curr Pain Headache Rep. 2010;14(6):418-423. doi:10.1007/s11916-010-0151-5

21. Ho KY, Gwee KA, Cheng YK, Yoon KH, Hee HT, Omar AR. Nonsteroidal anti-inflammatory drugs in chronic pain: implications of new data for clinical practice. J Pain Res. 2018;11:1937-1948. doi:10.2147/JPR.S168188

22. Jackson JL, Kuriyama A, Hayashino Y. Botulinum toxin A for prophylactic treatment of migraine and tension headaches in adults: a meta-analysis. JAMA. 2012;307(16):1736-1745. doi:10.1001/ jama.2012.505

23. Slavin KV, Nersesyan H, Wess C. Peripheral neurostimulation for treatment of intractable occipital neuralgia. Neurosurgery. 2006;58 (1):112-119. doi:10.1227/01.neu.0000192163.55428.62
Local and Regional Anesthesia

\section{Publish your work in this journal}

Local and Regional Anesthesia is an international, peer-reviewed, open access journal publishing on the development, pharmacology, delivery and targeting and clinical use of local and regional anesthetics and analgesics. The journal welcomes submitted papers covering original research, basic science, clinical studies, reviews \& evaluations, guidelines, expert opinion and commentary, case reports and extended reports. The manuscript management system is completely online and includes a very quick and fair peer-review system, which is all easy to use. Visit http://www.dovepress.com/testimonials. php to read real quotes from published authors. 